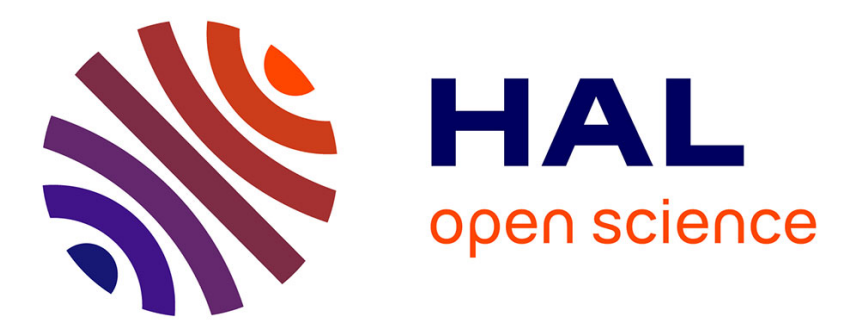

\title{
A Common Symmetrization Framework for Iterative (Linear) Maps \\ Alain Sarlette
}

\section{To cite this version:}

Alain Sarlette. A Common Symmetrization Framework for Iterative (Linear) Maps. Geometric Science of Information, Second International Conference, GSI 2015, Oct 2015, Palaiseau, France. 10.1007/9783-319-25040-3_73 . hal-01248787

\section{HAL Id: hal-01248787 \\ https://hal.inria.fr/hal-01248787}

Submitted on 28 Dec 2015

HAL is a multi-disciplinary open access archive for the deposit and dissemination of scientific research documents, whether they are published or not. The documents may come from teaching and research institutions in France or abroad, or from public or private research centers.
L'archive ouverte pluridisciplinaire HAL, est destinée au dépôt et à la diffusion de documents scientifiques de niveau recherche, publiés ou non, émanant des établissements d'enseignement et de recherche français ou étrangers, des laboratoires publics ou privés. 


\title{
A common symmetrization framework for iterative (linear) maps
}

\author{
Alain Sarlette \\ QUANTIC, Paris Sciences Lettres University, INRIA Rocquencourt, France
}

\begin{abstract}
This paper highlights some more examples of maps that follow a recently introduced "symmetrization" structure behind the average consensus algorithm. We review among others some generalized consensus settings and coordinate descent optimization.
\end{abstract}

\section{Introduction}

The linear consensus algorithm [1]

$$
x_{k}(t+1)=x_{k}(t)+\sum_{j=1}^{N} a_{j k}\left(x_{j}(t)-x_{k}(t)\right), \quad k=1,2, \ldots, N
$$

with $a_{j} k \geq 0$, or equivalently for $x_{k} \in \mathbb{R}, \quad x(t+1)=A(t) x(t) \quad$ where the matrix $A$ has off-diagonal components $A_{j k}=a_{j k}$ and diagonal component $A_{k k}=1-\sum_{j=1}^{N} a_{j k}$, features several geometric structures that could serve as a basis for generalization. In previous work we have considered the structure of (1) on a cone space, associated to the Hilbert metric, which generalizes (1) e.g. towards "non-commutative consensus" and the Kraus maps of quantum dynamics [2]. More recently [3], we have explored a structure related to the network interaction and generalized (1) into a "symmetrization" class of iterative maps, which is restricted to linear procedures but allows to cover consensus on (quantum) probability spaces and some geometric control design algorithms.

In the present paper, we want to give a few more examples of applications covered by the former approach. In particular:

- In Section 3 we show how symmetrization readily applies to the modeling of some direct variants of (1).

- In Section 4 we slightly relax the symmetrization assumptions to model gradient and coordinate descent (for a quadratic cost function to stay in the linear context).

The intended message of this paper is in showing the possibility to model a set of a priori different maps as one class of dynamics.

We denote by $I$ the identity matrix and by $e$ the identity element of a group; when numbering group elements we use the convention $g_{1}=e$. 


\section{Symmetrization}

Let us first recall the symmetrization framework developed in [3]. The main point is to write an iterative map

$$
x(t+1)=\Phi(x(t), t)
$$

as a stochastic combination of actions of a discrete group $\mathcal{G}$, i.e.

$$
\Phi(\cdot, t)=\sum_{i=1}^{|\mathcal{G}|} w_{i}(t) a\left(g_{i}, \cdot\right)
$$

where $g_{i} \in \mathcal{G}$ are the elements of the group, $a\left(g_{i}, \cdot\right)$ is a linear action associated to $g_{i}$ on the space $\mathcal{X} \ni x$, and the $w_{i}$ satisfy: $w_{i}(t) \geq 0$ for all $i, \sum_{i=1}^{|\mathcal{G}|} w_{i}(t)=1$.

The iterative map (2) can then be lifted, possibly non-uniquely, to dynamics on the stochastic weights of group elements. I.e. we can write

$$
x(t)=\sum_{i=1}^{|\mathcal{G}|} p_{i}(t) a\left(g_{i}, x(0)\right)
$$

and describe the evolution of the $p_{i}(t)$. Explicitly, defining $\pi_{i}(k)$ such that $g_{\pi_{i}(k)}=g_{i}^{-1} g_{k}$ in the group sense, we have

$$
p_{k}(t+1)=\sum_{i=1}^{|\mathcal{G}|} w_{i}(t) p_{\pi_{i}(k)}(t), \quad k=1,2, \ldots,|\mathcal{G}|,
$$

starting with $p_{1}(0)=1$ for $g_{1}=e$ and $p_{k}(0)=0$ for $k \neq 1$. In general, $|\mathcal{G}|$ can be much larger than the dimension of $\mathcal{X}$. Yet the interesting point is that (4) is completely independent of $\mathcal{X}$ and of the particular action $a\left(g_{i}, \cdot\right)$. This has allowed us to cover with one single model, consensus on variables belonging to vector spaces, so-called "symmetric consensus" on probabilities over a discrete set and similarly "symmetric quantum consensus". In the two latter applications, not only marginal probabilities but also all multi-partite correlation probabilities converge to the same values for all agents.

It is not difficult to see that the $|\mathcal{G}| \times|\mathcal{G}|$ state matrix describing (4) is doubly stochastic. It is hence stable and features as a particular stationary point the uniform weight distribution $p=\bar{p}$ where $\bar{p}_{k}=1 /|\mathcal{G}|$ for all $k$. For this stationary point, in the $\mathcal{X}$ space, we have

$$
\bar{x}=\frac{1}{|\mathcal{G}|} \sum_{i=1}^{|\mathcal{G}|} a\left(g_{i}, x(0)\right) .
$$

We hence call $\bar{x}$ the symmetrization of $x_{0}$ with respect to $(\mathcal{G}, a(\cdot, \cdot))$, and $a$ fortiori if $\Phi$ can be written as (3) then $\bar{x}$ is a stationary point of $\Phi$.

In general the goal of $\Phi$ is to make $x$ converge to $\bar{x}$. A sufficient condition for this is that the associated lift (4) makes $p$ converge to $\bar{p}$. In some situations, especially with time-varying $\Phi$, the lift might provide more insights, e.g.:

Proposition 1: Given $\delta>0, T>0$, denote $\mathcal{S}(t) \subseteq \mathcal{G}$ the set of $g_{i}$ for which $\sum_{t^{\prime}=t}^{t+T} w_{i}\left(t^{\prime}\right)>\delta$. If there exist $\delta>0, T>0$ and $\mathcal{H} \subseteq \mathcal{G}$ generating $\mathcal{G}$ such that - $g_{1}=e \in \mathcal{S}(t)$ for each $t$ and $\quad \mathcal{H} \subseteq \mathcal{S}(t)$ for each $t$, then the dynamics (4) makes $p$ converge to $\bar{p}$. 
With different choices of the lift, Proposition 1 can prove convergence of several different linear iterative maps at once, instead of having to repeat a "similar" proof argument for each specific map separately. In [3] a few examples are given. In the following we present a few others. Note that each of these examples has been efficiently solved on its own in the respective literature; our point here is that a common framework is established for all of them.

\section{$3 \quad$ Variations on consensus}

Let us first review how (1) can be cast into the symmetrization framework, at least for the usual case where $A$ is doubly stochastic, implying a.o. $\sum_{j} a_{j k} \leq 1$ for all $k$. Take $\overline{\mathcal{G}}=\operatorname{Perm}_{N}$ the group of permutations on $N$ elements. The easiest case is "gossip", where at each $t$ a single pair $(m, n)$ of agents interacts with $a_{m n}=a_{n m} \neq 0$ : we can then write $w_{1}=1-a_{m, n}$ for $g_{1}=e$ and $w_{i}=a_{m, n}$ for $g_{i}$ the pairwise permutation of $(m, n)$, all other $w_{i}$ zero. Several variations that allow several interactions at once, admit a similarly direct treatment.

For a general situation with $A$ doubly stochastic, we use a result by Birkhoff [4] to decompose $\Phi$ into a convex sum of permutations. If there exists $\alpha>0$ such that $a_{k k}>\alpha$ for all $k$, then $\tilde{A}=\frac{1}{1-\alpha}(A-\alpha I)$ is also doubly stochastic and by applying the Birkhoff result to $\tilde{A}$, we obtain a decomposition of $A=\alpha I+(1-\alpha) \tilde{A}$ into permutation matrices weighted by $w_{i}$ and where $w_{1} \geq \alpha$. Except for special time dependencies of the $a_{j k}(t)$, generally an element for which $w_{i}>\delta$ at some $t$ satisfies $\sum_{t^{\prime}=t}^{t+T} w_{i}\left(t^{\prime}\right)>\delta$ for all $t$, for $T$ large enough. We then say that all elements are recurrent. In this case, Proposition 1 ensures that $p(t)$ converges to the uniform distribution $\bar{p}$ over all elements of the group $\mathcal{G} \subseteq \overline{\mathcal{G}}$ generated by the elements that appear in the decomposition. Concretely, for instance:

- For pairwise gossip, if the interaction graph corresponding to the $\bar{a}_{j k}=$ $\sum_{t^{\prime}=t}^{t+T} a_{j k}\left(t^{\prime}\right)$ is connected, then the associated pairwise permutations generate all of $\overline{\mathcal{G}}=\operatorname{Perm}_{N}$. A generalization to the case where the interaction graph is composed of several connected components is immediate.

- When $a_{j k}=\beta / N$ for all $j \neq k$ and some $\beta \in(0,1)$, an associated lift would be $w_{1}=1-\beta$ and $w_{i}=\beta / N$ for all $g_{i}$ which are a power 1 to $N-1$ of the circular permutation $g_{c}:(1,2,3, \ldots, N) \rightarrow(N, 1,2, \ldots, N-1)$. These form a closed subgroup $\mathcal{G} \subset \operatorname{Perm}_{N}$. But still, having $p$ converge to the uniform distribution over these $N$ elements is sufficient to have the $x_{k}$ converge to $1 / N \sum_{k=1}^{N} x_{k}(0)$ for each $k$. Problems with the lift could arise if an element of $\operatorname{Perm}_{N} \backslash \mathcal{G}$ appears rarely, i.e. without belonging to the set $\mathcal{S}(t)$ of Proposition 1 for any large $T$. This seems unlikely in practical situations.

In this setting, whether $x_{k}$ is a scalar, vector or other object of a linear space $\mathcal{X}$ does not matter, it just affects the group actions. Another action allows to apply the same group dynamics to the symmetrization of joint probability distributions, both classical and quantum, over a finite set [3]. We now turn to different variants of (1) for $x_{k} \in \mathbb{R}$. 


\subsection{Consensus with antagonistic interactions}

A variant of consensus proposed e.g. in [5] considers that agents might be attracted to the opposite of the values of some neighbors, i.e. the dynamics become

$$
x_{k}(t+1)=a_{k k} x_{k}(t)+a_{j k} x_{j}(t)
$$

with $a_{k k}=1-\sum_{j \neq k}\left|a_{j k}\right|>0$, but possibly some negative $a_{j k}$ for $j \neq k$.

To cover this possibility, we take $\tilde{\mathcal{G}}$ the group corresponding to all permutation matrices with arbitrary sign \pm 1 on each component; we call this "generalized" permutations. This can be viewed as a particular product of the group of permutations with the group $\{-1,1\}^{N}$. We then lift (5) as follows.

- Take the Birkhoff decomposition associated to the corresponding standard consensus algorithm, where each $a_{j k}$ in (5) is replaced by $\left|a_{j k}\right|$. This gives nonzero weights $\tilde{w}_{i}$ on the $g_{i} \in \mathcal{G}$ corresponding to permutation matrices.

- Then sequentially consider each pair $(j, k)$ for which $a_{j k}<0$ and swap the weights attributed to the generalized permutation matrices which are equal up to the sign of their element $(j, k)$.

Convergence of (5) is efficiently characterized a.o. in [6].We just provide an informal summary on the basis of $\tilde{\mathcal{G}}$. As for standard consensus, we may generally assume that all the elements appearing in the lift are recurrent. The generated group $\mathcal{G}$ over which we get $p=\bar{p}$ at convergence then depends (only) on the group generated by the appearing elements. Three main situations can occur:

- The interaction graph is not connected.

- The component $(k, k)$ takes the same sign in the matrices associated to all the generated group elements. By group properties, this implies that all components $(j, j)$ have the same sign in all the generated group elements, provided the interaction graph is connected. Then in terms of $x_{k}$, the agents split into two communities with opposite component values as $t \rightarrow \infty$.

- The component $(k, k)$ can take opposite signs in the generated group elements. Then, thanks to group properties, for each $j, k$ there should be an equal number of elements with negative component $(j, k)$ as with positive component $(j, k)$ in the generated group $\mathcal{G}$. This implies that when $p$ converges to $\bar{p}$ uniform over $\mathcal{G}$, the corresponding $x_{k}$ converge to 0 .

Our lift can involve many elements but still a finite number, i.e. it abstracts away the exact values of the $a_{j, k}(t)$ in (5). While our framework may appear more cumbersome than the graph analysis of [6], it can also yield more precise results in some cases. Indeed, we can exactly cover the following example which [6] provides as an inconclusive case of their graph-conditions-based theorem:

$$
\begin{aligned}
& x_{k}(t+1)=1 / 2 \quad\left(x_{1}(t)+x_{2}(t)\right), \quad k=1,2 ; \quad t \text { even } \\
& x_{k}(t+1)=(-1)^{k} / 2 \quad\left(x_{2}(t)-x_{1}(t)\right), \quad k=1,2 ; \quad t \text { uneven } .
\end{aligned}
$$

The dynamics can be explicitly solved and converges to $x_{1}=x_{2}=0$ after iterations $t=0, t=1$, yet the theorem of [6] cannot tell this. In terms of our 
lift, we have three recurrent elements

$$
g_{1}=\left(\begin{array}{ll}
1 & 0 \\
0 & 1
\end{array}\right) ; g_{2}=\left(\begin{array}{ll}
0 & 1 \\
1 & 0
\end{array}\right) ; g_{3}=\left(\begin{array}{cc}
0 & -1 \\
-1 & 0
\end{array}\right)
$$

with weights $w_{1}=1 / 2, w_{2}(t)=1 / 4+(-1 / 4)^{t}, w_{3}(t)=1 / 4-(-1 / 4)^{t}$. These weights are in fact irrelevant, we only need to compute that $g_{1}, g_{2}, g_{3}$ generates a 4 -group element, together with $g_{4}=-g_{1}$. We then readily conclude that the system converges to $\bar{x}=\frac{1}{4} \sum_{i=1}^{4} g_{i} x=0$.

At this stage we do not claim that the group lift approach is efficiently scalable to larger problems. Yet it might suggest e.g. how to adapt the graph analysis argument of [6] towards covering the above example.

\subsection{Doubly sub-stochastic matrices}

Consensus is a particular linear dynamics which features a one-dimensional invariant space. In iterative maps, the most common situation is when there is only one target invariant point. This happens with "smaller than stochastic" $A$. Shifting the invariant point to $x=0$, we define the following class of systems:

$$
\begin{aligned}
& x_{k}(t+1)=\sum_{j=1}^{N} a_{j k}(t) x_{j}(t) \text { with } \quad 1 \geq \sum_{j}\left|a_{j k}(t)\right| \text { for all } k, t \\
& 1 \geq \sum_{k}\left|a_{j k}(t)\right| \text { for all } j, t .
\end{aligned}
$$

Such system can be straightforwardly rewritten as consensus with (possibly antagonistic interactions and) a virtual leader $x_{0}(t)=0$, hence proving convergence is not a big issue. Again our intended contribution is more to show that this dynamics, as well, can be cast into the class of symmetrization algorithms. For simplicity of notations we describe the following assuming $a_{j k} \geq 0$ for all $j, k$.

Take the same group $\tilde{\mathcal{G}}$ as in Section 3.1. We first define $b_{j k}=a_{j k}+c_{j k}$ with $c_{j k} \geq 0$ and such that $\sum_{j=1}^{N} b_{j k}=\sum_{j=1}^{N} b_{k j}=1$ for all $k$, i.e. the state matrix associated to the $b_{j k}$ is doubly stochastic. Such a construction is always possible for a sub-stochastic matrix, e.g. as follows:

0 . Let $a_{j k}^{\prime}:=a_{j k}$ for all $j, k$.

1. Compute $a_{k}^{c}=\sum_{j=1}^{N} a_{j k}^{\prime}$ and $a_{k}^{r}=\sum_{j=1}^{N} a_{k j}^{\prime}$ for all $k$.

2. Since $\sum_{k} a_{k}^{c}=\sum_{j, k}^{j} a_{j k}^{\prime}=\sum_{k} a_{k}^{r}$ : if $a_{k}^{c}<1$ for some $k$ then there exists $k^{\prime}$ such that $a_{k^{\prime}}^{r}<1$, and vice versa. Else $a_{k}^{c}=a_{k}^{r}=1$ for all $k$, i.e. the $a_{j k}^{\prime}$ matrix is already doubly stochastic and we can stop.

3. Find $a_{m}^{r}=\max \left(a_{k}^{r}: a_{k}^{r}<1\right)$ and $a_{n}^{c}=\max \left(a_{k}^{c}: a_{k}^{c}<1\right)$. Then let $b_{m n}=$ $a_{m n}^{\prime}+c_{m n}$ with $c_{m n}=1-\max \left(a_{m}^{r}, a_{n}^{c}\right)$, and $b_{j k}=a_{j k}^{\prime}$ for all $(j, k) \neq(m, n)$.

4. Define $a_{j k}^{\prime}:=b_{j k}$ for all $j, k$ and iterate the procedure starting again from 1 . This procedure converges to a doubly stochastic matrix. Indeed, at each iteration: if the $a_{j k}^{\prime}$ are doubly sub-stochastic then also the $b_{j k}$ are; and the $b_{j k}$ matrix has at least one more row or column whose elements sum to 1 than the $a_{j k}^{\prime}$ matrix.

Define by $\left\{\tilde{w}_{i}\right\}$ a lift of the dynamics associated to the standard consensus $x_{k}(t+1)=\sum_{j=1}^{N} b_{j k}(t) x_{j}(t)$. We then sequentially go through all $(j, k)$ for which $c_{j k}>0$ and transform the weights as follows. 
0 . Let $w_{i}^{\prime}=\tilde{w}_{i}$ for all $i$ and choose some $(j, k)$ for which $c_{j k}>0$.

1. Denote $f_{j k}^{-}(m)=n$ such that $g_{n}$ is identical to $g_{m}$ except that the matrix element $(j, k)$ associated to $g_{m}$ has an opposite sign in $g_{n}$. Then for all $i$ for which $g_{i}$ has a positive element $(j, k)$, apply

$$
w_{i}=\left(1-\frac{c_{j k}}{2 b_{j k}}\right) w_{i}^{\prime}, \quad w_{f_{j k}^{-}(i)}=\frac{c_{j k}}{2 b_{j k}} w_{i}^{\prime} .
$$

2. Let $w_{i}^{\prime}=w_{i}$, choose a different $(j, k)$ with $c_{j k}>0$ and iterate from point 1 .

The weight modification in point 1 above preserves the total weight, $\sum_{i} w_{i}=$ $\sum_{i} w_{i}^{\prime}$. The $w_{i}$ remain non-negative, since $c_{j k} \geq 0, b_{j k} \geq 0, a_{j k} \geq 0$ and $1-\frac{c_{j k}}{2 b_{j k}}=$ $1-\frac{c_{j k}}{2 c_{j k}+2 a_{j k}}>0$. Summing up the effect on $\sum_{i} w_{i} a\left(g_{i}, \cdot\right)$, we see that when point 1 treats $(j, k)$, the matrix components different from $(j, k)$ undergo no change while the component on $(j, k)$ is modified:

$$
\text { from } \sum_{i \in \mathcal{F}_{j, k}} w_{i}^{\prime}=: B_{1} \quad \text { to } \quad \sum_{i \in \mathcal{F}_{j, k}} w_{i}^{\prime}\left(\left(1-\frac{c_{j k}}{2 b_{j k}}\right)-\frac{c_{j k}}{2 b_{j k}}\right)=: B_{2},
$$

where $\mathcal{F}_{j, k} \subset \tilde{\mathcal{G}}$ is the subset of group elements whose associated matrix have a coefficient +1 at position $(j, k)$. By definition of the lift, we have $B_{1}=b_{j k}$. We then get $B_{2}=B_{1}\left(1-\frac{c_{j k}}{b_{j k}}\right)=a_{j k}$, thus indeed the matrix that we had to model.

From there, we can go on and analyze convergence of the resulting group dynamics. By construction, if $\mathcal{G}$ contains $g_{i}$ then also contains $g_{f_{j k}^{-}(i)}$ for each $(j, k)$ for which $c_{j k}>0$. Hence if $p(t)$ converges to $\bar{p}$ uniform over $\mathcal{G}$, then the corresponding $(j, k)$ elements will be zero in the matrix associated to $\frac{1}{|G|} \sum_{i=1}^{|\mathcal{G}|} a\left(g_{i}, \cdot\right)$.

\section{Gradient Descent and Coordinate Descent}

In optimization, the gradient descent method consists in iteratively searching for min $f(x)$ by applying

$$
x(t+1)=x(t)-\alpha \operatorname{grad}_{x} f(x(t)) \quad, \quad \alpha>0, \quad x \in \mathbb{R}^{N} .
$$

For a local quadratic expansion $f=\frac{1}{2} x^{T} A x$ around the minimum, $A$ symmetric positive definite, approximating (7) by a linear map, there is a trivial symmetrization viewpoint on the resulting $x(t+1)=x(t)-\alpha A x(t)$.

In the eigenbasis of $A,(7)$ becomes $x_{k}(t+1)=\left(1-\alpha a_{k}\right) x_{k}(t)$ with $a_{k}$ the associated eigenvalues. For stable algorithms, $\left(1-\alpha a_{k}\right) \in(-1,1)$ such that the associated state transition matrix is (diagonal and) doubly substochastic. A simplified version of section 3.2 then rewrites this iteration as symmetrization over the group $\mathcal{G}$ represented by diagonal $N \times N$ matrices with elements \pm 1 . This abstract definition does not require to actually compute the eigenbasis only assume it exists and the $a_{k}$ are small enough. Yet this viewpoint must be adapted to address the following. 


\subsection{Coordinate Descent}

Coordinate descent selects some $k \in\{1,2, \ldots, N\}$ at each step and applies gradient descent to minimize $f(x)$ along a line where only $x_{k}$ varies, assuming fixed all $x_{j}$ with $j \neq k$. This comes closer to a (possibly stochastically) time-varying map as in consensus. It links to symmetrization as follows, e.g. for $N=2$ : define

$$
y=\left(\begin{array}{cc}
\sqrt{a_{1}} & 0 \\
0 & \sqrt{a_{2}}
\end{array}\right)\left(\begin{array}{cc}
\cos \theta & \sin \theta \\
-\sin \theta & \cos \theta
\end{array}\right) x
$$

such that $f(x)=\frac{1}{2} x^{T} A x=\frac{1}{2} y^{T} y$. This simplifies the cost function, but coordinate descent now implies iterative optimization along two arbitrary lines, of slopes $\tan \phi_{1}=\tan \theta a_{2} / a_{1}$ and $\tan \phi_{2}=-\operatorname{cotan} \theta a_{2} / a_{1}$. In general those lines are not orthogonal, but $f$ will still be quadratic along each of them. It is not difficult to see that we can then write, if $k \in\{1,2\}$ is chosen at time $t$ :

$$
\begin{aligned}
y(t+1) & =(1-\lambda) y(t)+\lambda\left(\text { reflection of } y(t) \text { around axis of slope } \operatorname{cotan} \phi_{k}\right) \\
& =(1-\lambda) y(t)+\lambda\left(\begin{array}{cc}
-\cos 2 \phi_{k} & \sin 2 \phi_{k} \\
\sin 2 \phi_{k} & \cos 2 \phi_{k}
\end{array}\right) y(t),
\end{aligned}
$$

for some $\lambda \in(0,1)$. In general, $(1-\lambda)+\lambda(\cos +\sin )>1$ and the associated transition matrix is not sub-stochastic. Nevertheless, we were able to write this as symmetrization with respect to the 8-element group $\mathcal{G}=$

$$
\left\{g_{1}^{ \pm}= \pm\left(\begin{array}{ll}
1 & 0 \\
0 & 1
\end{array}\right), g_{2}^{ \pm}= \pm\left(\begin{array}{cc}
1 & 0 \\
0 & -1
\end{array}\right), g_{3}^{ \pm}= \pm\left(\begin{array}{ll}
0 & 1 \\
1 & 0
\end{array}\right), g_{4}^{ \pm}= \pm\left(\begin{array}{cc}
0 & 1 \\
-1 & 0
\end{array}\right)\right\} .
$$

Explicitly, $y(t+1)=\left(w_{1+} g_{1}^{+}+w_{2-} g_{2}^{-}+w_{2+} g_{2}^{+}+w_{3-} g_{3}^{-}+w_{3+} g_{3}^{+}\right) y(t)$ with

$$
\begin{array}{lll}
w_{1+}=1-\lambda ; & w_{2-}=\lambda\left(\frac{1}{4}+\frac{\cos \phi_{k}}{2}\right) ; \quad w_{3+}=\lambda\left(\frac{1}{4}+\frac{\sin \phi_{k}}{2}\right) \\
& w_{2+}=\lambda\left(\frac{1}{4}-\frac{\cos \phi_{k}}{2}\right) ; \quad w_{3-}=\lambda\left(\frac{1}{4}-\frac{\sin \phi_{k}}{2}\right) .
\end{array}
$$

By construction $\sum_{g_{i} \in \mathcal{G}} w_{i}=1$. However we do not have $w_{i}>0$ for all $i$. This requires to slightly generalize the class of dynamics accepted on the group. In the present case:

The state transition matrix for the weights $p_{i}(t)$ on the group $\mathcal{G}$ is symmetric and doubly stochastic, up to having possibly negative off-diagonal components. Hence it still features $\bar{p}$ as a stationary point. Its convergence is not as straightforward to analyze as standard consensus. However thanks to symmetry, in a time-varying context it suffices for convergence to examine the common Lyapunov function $V(t)=\sum_{i}\left(p_{i}(t)\right)^{2}$.

For this particular example, i.e. a two-dimensional $x$, the state transition matrices for the $p_{i}(t)$ further admit an efficient tensor product decomposition such that convergence conditions can be easily formalized. At this point we cannot claim that the symmetrization framework is efficient to study convergence for high-dimensional $x$. Yet, the possibility to model the system as symmetrization, with generalized (possibly negative) weights, could suggest a way to include coordinate descent in versatile theoretical formalizations that exploit the symmetrization structure. 


\section{Conclusion}

We have abstractly defined a "symmetrization" class of linear iterative maps. This class comprises a great variety of algorithms, as illustrated in [3]. The present paper highlights some further elements of the class. Although each instance encountered so far, admits a relatively easy specific convergence proof, the common symmetrization framework allows to treat them all at once, modulo a finite group analysis step. In the future we hope that further properties of this symmetrization class can be exploited in general analyzes.

\section{Acknowledgments}

I thank F.Ticozzi and L.Mazzarella for sharing ideas on the symmetrization approach and R.Sepulchre for encouraging to address these particular applications.

\section{References}

1. J.N. Tsitsiklis, Problems in decentralized decision making and computation, $\mathrm{PhD}$ Thesis, MIT, 1984.

2. R. Sepulchre, A. Sarlette and P. Rouchon, Consensus in non-commutative spaces, Proc. 49th IEEE Conf. on Decision and Control, pp. 5596-6601, 2010.

3. L. Mazzarella, A. Sarlette and F. Ticozzi, Extending robustness and randomization from consensus to symmetrization algorithms, SIAM J. Control \& Optimization, in press; see http://arxiv.org/abs/1311.3364, 2014.

4. G. Birkhoff, Three observations on linear algebra, Univ. Nac. Tucuan. Revista A vol. 5, pp. 147-151, 1946.

5. Z. Meng, G. Shi, K.-H. Johansson, M. Cao, and H. Yiguang, Modulus consensus over networks with antagonistic interactions and switching topologies, preprint http://arxiv.org/abs/1402.2766, 2014.

6. J. Hendrickx, A Lifting Approach to Models of Opinion Dynamics with Antagonisms, Proc. 53rd IEEE Conf. on Decision and Control, 2014. 